\title{
The Effect of Different Surface Modifications on Titanium Dental Implant Surface Characteristics and Bacterial Adhesion
}

\author{
Afya Sahib Diab Al-Radha; ${ }^{1}$ BDS; MSc; \\ MFDSRCPS (Glasgow), PhD (Uk); \\ Dr. Charles Younes, MSC, PhD, Doctorate es-Sciences (Paris XI)2;
}

\author{
Dr. Peter Heard, MA, PhD (Cantab) ${ }^{3}$ Prof. Howard F. Jenkinson, BSc, PhD4 \\ ${ }^{1}$ Lecturer in Oral and Maxillofacial Surgery Department; College of Dentistry, \\ Al- Mustansiriyah University, Baghdad, Iraq. \\ ${ }^{2}$ Senior Research Fellow, Interface Analysis Centre, University of Bristol, Bristol, United Kingdom. \\ ${ }^{3}$ Research Fellow, Interface Analysis Centre, University of Bristol, Bristol, United Kingdom \\ 4Professor, School of Oral and Dental Sciences, University of Bristol, Bristol, United Kingdom.
}

\begin{abstract}
Purpose: The presented work was to evaluate the effect of different surface modifications on the physiochemical characteristics of titanium surfaces, and to examine the influence of these modifications on bacteria adhesion with and without saliva coating.

Materials and Methods: Pure polished titanium discs were subjected to various surface treatments. Surface topography was examined. Contact angle, surface free energy, crystalline phase, chemical composition, roughness, and microhardness were also evaluated.

Titanium discs were incubated with a suspension of each of the chosen bacteria for 6 hours and the percentage of area covered by the bacteria was calculated using fluorescence microscope images.

Results: A significant difference was found in contact angle measurements with a wide variation in most of the measured physiochemical properties between the modified surfaces. A significant reduction was observed in bacterial adhesion to surfaces with high surface hydrophobicity and low surface free energy after coating with saliva.

Conclusions: Physiochemical properties of titanium can be altered depending on the type of modification, and most of the modifications to a titanium surface increase its hydrophobicity and decrease its surface free energy. Increased surface hydrophobicity along with decreased surface free energy leads to decrease bacterial adhesion to titanium surface after coating with saliva.
\end{abstract}

Keyword: Dental implant, bacteria, titanium, surface modification, physiochemical characteristics, surface free energy

\section{Introduction}

Dental implants have become an important option to replace missing teeth. However, implant failure and peri-implant disease is still a problem facing implantologists. ${ }^{1}$

Many efforts have been made to develop implant surfaces that can accelerate osseointegration, ${ }^{2}$ enhance gingival attachment to provide a soft tissue seal that prevents bacterial invasion, ${ }^{3}$ decrease bacterial adhesion and/or has bactericidal effects. ${ }^{4}$

Topography of the biomaterial surface plays an important role in determining the cellular response. ${ }^{5}$ The biological responses to a dental implant are determined by a number of physical and chemical features of the implant surface: chemical composition, crystalline phase, surface wettability, surface energy, and surface topography. ${ }^{6}$ Any biological interaction with the implant material will be influenced by these properties and any changes in one of these groups will affect the other parameters. ${ }^{7,8,9}$

Different surface treatments have been applied to improve the surface topography and chemical composition of the implant surface, ${ }^{10}$ which has resulted in high clinical osseointegration success rates. However, the absorption of biomolecule pellicles and the subsequent growth of bacteria on these surfaces is still one of the stimuli for the introduction of peri-implant inflammatory processes. Therefore, it is advantageous to modify titanium surfaces to inhibit the initial adhesion of bacteria and produce a surface which is plaque resistant and which, in turn, may prevent peri-implantitis or limit its progression. ${ }^{4}$ 
It is important to investigate the relationship between different surface properties and bacterial adhesion. Despite the large number of studies about the biological response to dental implants, it is still not well known which specific surface characteristics affect the microbial response which necessitates the need for further studies.

The research hypotheses were: (a) Physiochemical properties of titanium surface altered differently according to each type of surface modification. (b) Precise physiochemical properties of titanium surface affect bacterial adhesion.

The aim of the work presented herein was to investigate the influence of different titanium surface modifications on the physiochemical characteristics of these surfaces, and to examine the effect of these modified titanium surfaces on bacterial adhesion. Wide range of modified titanium surfaces with known surface characteristics have been investigated in order to determine the key metal-properties that influence microbial adhesion.

\section{Materials and Methods}

Specimens Preparation and surface modification Disc shaped specimens, about $5 \mathrm{~mm}$ in diameter, were punched from annealed titanium sheets $(99.6+\%)$ (Goodfellow, Cambridge Limited, England). In order to achieve an accurate evaluation of surface changes and a proper comparison between the groups of specimens, a clean mirror finish surface was prepared by mechanical grinding and polishing on one side of each disc.

The surface of the mechanically polished titanium discs, labeled as PT, were modified using different procedures: Etching with $0.5 \mathrm{wt} \%$ Hydrofluoric acid at room temperature for $1.5 \mathrm{~min}(\mathrm{HF})$; Dual acid etching with $69 \% \mathrm{H}_{2} \mathrm{SO}_{4}$ and $37 \% \mathrm{HCl}$ at $80^{\circ} \mathrm{C}$ at a ratio of 1:1 for $1.5 \mathrm{~min}(\mathrm{DA})$. After etching, the discs were rinsed immediately with distilled water. In addition, a chemical etch with alkali followed by a heat treatment (AEHT) were performed on a group of specimens. These were first immersed in a $5 \mathrm{M}$ sodium hydroxide $(\mathrm{NaOH})$ solution maintained at $60{ }^{\circ} \mathrm{C}$ in a water bath for 24 hours then rinsed with distilled water and dried and subsequently heat treated at $600{ }^{\circ} \mathrm{C}$ for $1 \mathrm{~h}^{11}$ The heat treated (HT) titanium discs were exposed to a temperature of $750{ }^{\circ} \mathrm{C}$ for 1 hour. ${ }^{12}$

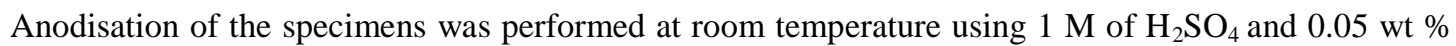
hydrofluoric acid as an electrolyte by applying a potential of 80 Volts for 5 minutes. The disc-specimen was connected individually to the anode with only the specimen surface exposed to the electrolyte to ensure a constant surface area ratio which was 1:10 between anode and cathode. The cathode was made from a platinum sheet. The distance between cathode and anode was $\sim 3 \mathrm{~cm}$. During the period between preparation and analysis, the specimens were kept in an air tight container.

\section{Surface analysis}

Surface roughness measurements were conducted using non contact optical Proscan profilometry (Proscan 2000, Scantron Industrial Products Ltd., England). Ra was calculated for each sample "Ra: the arithmetic mean of the absolute values of the surface point departures from the mean plane within the sampling area". 13

Topographic inspection was conducted using scanning electron microscopy (SEM) (A PC-controlled ISI 60 SEM) and light microscopy (Olympus PH2). The wettability of discs was calculated by assessing static water contact angles with "DSA 100" contact angle measuring system (KRUSS Advancing Surface Science. $\mathrm{GmbH}$ ) using the sessile drop technique. The measurements were carried out at body temperature $37^{\circ} \mathrm{C}$ with distilled water as the probe liquid. Surface free energy (SFE) was measured using the contact angle measurement of three wetting agents: Water, Ethylene glycol, Diiodo-Methane. This calculation was conducted using the concept of polar and dispersion components using Owen's method for calculation. ${ }^{14}$ Highly specialized drop shape analysis software was used to conduct this measurement (SOFTWARE - DSA3).

Raman spectrometry was used to characterize surface crystallinity. A Raman system (Renishaw Ramascope 2000) equipped with a He-Ne laser (wavelength $633 \mathrm{~nm}$ ) was operated with a $25 \mathrm{~mW}$ laser power and $4 \mu \mathrm{m}$ diameter laser spot impinging on the specimen surface. A $100-1000 \mathrm{~cm}^{-}{ }^{1}$ wave number range with 3 $\mathrm{mW}$ laser power and acquisition time of $10 \mathrm{sec}$. were used to acquire each spectrum.

Microhardness tests were conducted using a diamond pyramid indenter procedure with a MNIN LOAD hardness tester (Erust Leitz, Wetzlar, Germany). A 500g load was applied by the diamond indenter for $15 \mathrm{sec}$. The test was done on a microscopic scale.

Chemical measurements were conducted using X-ray Photoelectron Spectroscopy (XPS)

(Perkin-Elmer XPS system model 10-360) equipped with Omni Focus ${ }^{\mathrm{TM}}$ lenses. The sample was irradiated with a monoenergetic soft X-ray Al Kó $(1486.6 \mathrm{eV})$ in an ultrahigh vacuum $\left(<10^{-8}\right.$ torr $)$. The element chemical composition analyzed surfaces were measured using the VGS5250 software.

\section{Saliva preparation:}


Saliva was collected from 9 healthy donors in ice chilled tubes early in the morning then treated with $1 \%$ of 0.25 Dithiothreitol (DTT) (Sigma) before centrifugation at $12000 \mathrm{rpm}$ for $10 \mathrm{~min}$ at $4{ }^{\circ} \mathrm{C}$. The supernatant was diluted to $50 \%$ and then filter sterilized by passing through $0.22 \mu \mathrm{m}$ a cellulose nitrate membrane filter (Sarstedt Inc, USA). The sterile saliva was stored at $-20{ }^{\circ} \mathrm{C}$ and defrosted immediately before use.

Titanium discs were incubated with saliva for 2 hours at $37{ }^{\circ} \mathrm{C}$ to coat with saliva, then gently rinsed to remove excess saliva.

\section{Bacteriology}

Pure cultures of clinically isolated bacteria were used in the study, and bacterial identity was confirmed using 16 rDNA gene sequences, gram stain and microscopical imaging. The resulting isolates were purified and frozen in $-70{ }^{\circ} \mathrm{C}$ as stocks.

Streptoccocus mitis was cultured from frozen stocks and plated on Blood agar base No. 2 (LAB) supplemented with $5 \%$ defibrinated horse blood (CS-Bioscience LTD) and grown at $37{ }^{\circ} \mathrm{C}$ aerobically in $\mathrm{O}_{2}$ depleted atmosphere "candle jar".

Fusobacterium nucleatum was cultured from frozen and plated onto Fastidious Anaerobic Agar plates (FAA; LAB M) containing $5 \%$ defibrinated horse blood (C S-Bioscience LTD) and grown at $37{ }^{\circ} \mathrm{C}$ in an anaerobic cabinet (Don Whitley Scientific) in an atmosphere containing $10 \%$ hydrogen, $10 \%$ carbon dioxide and $80 \%$ nitrogen.

For adhesion experiments S. mitis was grown overnight in BHY broth $\{3.7 \%$ Brain heart infusion broth $(\mathrm{LAB} M)+0.5 \%$ Yeast extract $\left(\right.$ Bacto $^{\mathrm{Tm}}$ ) in an $\mathrm{O}_{2}$ depleted atmosphere "candle jar". Fastidious anaerobic broth (FAB; LAB M) was used for culture of $\mathrm{F}$. nucleatum and incubated at $37{ }^{\circ} \mathrm{C}$ in an anaerobic cabinet for two days. Bacteria were harvested by centrifugation at $2500 \mathrm{rpm}$ for 15 minutes. Pellet were then resuspended in $5 \mathrm{ml}$ of Tris $\mathrm{HCl}$ buffer ( $\mathrm{pH} \mathrm{7.2)}$ and washed and re-harvested twice. Finally, bacteria were resuspended in the same buffer and density adjusted to $75^{*} 10^{7}$ colony forming unites $/ \mathrm{ml}$. using a $600 \mathrm{~nm}$ spectrophotometer to measure the optical density (O.D.) as calculated against standards for each bacterial strain. Five discs for each surface were incubated with $2 \mathrm{ml}$ of suspension of each bacteria type separately in sterile cell culture containers (Costar, 12 well cell culture clusters). This was done at $37{ }^{\circ} \mathrm{C}$ for 6 hours with gentle rotation with the modified surface of the discs placed facing upward. After the incubation period, discs were gently rinsed 5 times for 1 minute (to remove non-attached bacteria) and then fixed with $2 \mathrm{ml} 2.5 \%$ gluteraldehyde (Sigma-Aldrich) for $30 \mathrm{~min}$ on ice, followed by an additional fixation step with $1.5 \%$ FITC (fluorescein isothiocyanate, isomer I, Sigma) at room temperature for $30 \mathrm{~min}$. Discs were then washed to remove excess dye. Two experiments were conducted on each bacterium, one with saliva coating and the other without. Each experiment was repeated twice on a different day.

\section{Bacterial adhesion assays}

Fluorescence microscopy (Leica DM LB) was used to obtain images for counting FITC-stained bacteria attached to surfaces. Each disc was examined at 100-fold magnification under oil immersion $(100 * / 1.30$ oil) and five digital images per disc were taken from different areas on each surface.

Percentage of area covered by bacteria was calculated using Image J software (http://rsb.info.nih.gov/ij) for a fixed surface area. Experimental procedures were conducted blindly.

\section{Statistics}

Statistical analysis was carried out using Excel and STAT GRAPHICS plus 5.0 programs.

Methods used to analyze and assess results were descriptive statistics: Mean, standard deviation, standard error. Kruskal-Wallis test was used to test the significancy with in each group. Mann-Whitney test was used to determine to determine the specific differences between the group members.

The probability value ( $\mathrm{p}$-value) considered significant at $\mathrm{P}<0.05$ and highly significant if $\mathrm{P}<0.01$.

\section{Characteristics of surface topography}

\section{Results}

Surface roughness was measured by profilometry and the mean surface roughness values obtained for the surface modifications are listed in Table 1. All the surfaces have Ra value less than $0.2 \mu \mathrm{m}$ in which the PT surface showed the lowest Ra value with highly significant differences with all other surfaces $(\mathrm{P}<0.01)$. A significant difference in surface roughness $(\mathrm{Ra})$ was found between HT and DA surfaces $(\mathrm{P}=0.019)$.

Topography of the different surfaces was revealed by scanning electron microscopy, as shown in Figure 1. Each surface modification has its own effect on the polished titanium surface. In the AN surface specific pits shape can be seen, the HF surface showed clear distinguished grain boundaries. DA surface showed the characteristic feature of acid etching with pits and scratches across the whole surface. The HT surface showed the creation of 'hills and valleys' across the surface. However, for the PT and AEHT surfaces no obvious changes were observed. 
Optical microscopic images (Figure 1) showed details that were not revealed by SEM images. The exposed grain was so obvious in the DA surface in a manner which differed from that of the HF surface. These grains were not observed on the PT surface. Colored images seen for both the AN and AEHT surfaces showed mixture of many colors which did not precisely reflect colors seen by the naked eye.

Microhardness results can be seen in Table 1. The HT surface showed more than one hundred Vickers point number (VPN) increase in microhardness when compared with the original control PT with highly significant differences $(\mathrm{P}<0.01)$ to all titanium surfaces. Similarly, both AEHT and DA surfaces had significant differences when compared with the $\mathrm{PT}$ surface $(\mathrm{P}=0.028 ; 0.0281$ respectively) and highly significant differences with both the $\mathrm{AN}$ and the HF surface $(\mathrm{P}<0.01)$.

Titanium surface wettability was changed significantly using different modification procedures, as shown in Table 2 . The results of surface wettability can be organized into three sets of paired surfaces with each set showing highly significant differences $(\mathrm{P}<0.01)$ to all of the other surfaces but not between themselves. Both the PT and HT surfaces showed the lowest values of contact angle measurement followed by the AN and AEHT surfaces with increase in contact angle values. The most hydrophobic surfaces were both the HF and DA surfaces with the highest contact angle values.

Apart from the anodization procedure that led to an increase in SFE compared with the original control PT surface; it can be noted that all other surface modifications of the titanium surface led to a reduction in their SFE at different levels.

PT, DA and HF surfaces showed amorphous oxide layer, while AN surface had anatase crystalline oxide layer. Both HT and AEHT surface showed rutile crystalline oxide layer.

The major chemical constituents of all surfaces were (titanium, oxygen, carbon), some surface modifications exhibited traces of other elements beside these major component elements. For the AEHT surface calcium was detected. On the HF and AN surfaces fluoride was found in addition to nitrogen on the AN surface

\section{Bacteriology}

Adhesion of bacteria to different surfaces is shown in Table 3. The mean percentage of area covered by $S$. mitis did not show any significant difference between the modified titanium surfaces without saliva coating $(\mathrm{P}=0.09)$. Coating these surfaces with saliva resulted in highly significant differences between the surfaces $(\mathrm{P}<0.01)$ for $S$. mitis adhesion.

The percentage of $S$. mitis adhesion increased on the HT surface after saliva coating with highly significant differences to all modified titanium surfaces $(\mathrm{P}<0.01)$ but not with the PT surface. Conversely, the HF surface showed the lowest percentage of bacterial adhesion with significant differences to the AN surface $(\mathrm{P}=0.035)$ and highly significant differences $(\mathrm{P}<0.01)$ to all other modified titanium surfaces. Highly significant differences $(\mathrm{P}<0.01)$ within each modified titanium surface were found between the percentage of bacterial adhesion to the same surface with and without saliva coating.

Both the HT and AEHT surfaces had the highest percentages of $F$. nucleatum adhesion, showing highly significant differences to all other surfaces $(\mathrm{P}<0.01)$ except with the AN surface. The AN surface had a significant difference with the HT surface $(\mathrm{P}=0.032)$ and had highly significant differences with the PT, DA and HF surfaces $(\mathrm{P}<0.01)$.

However, coating the surfaces with saliva led to an increase in percentage of $F$. nucleatum adhesion to the PT surface with significant difference with AEHT $(\mathrm{P}=0.029)$ and highly significant differences with both the HF and DA surfaces $(\mathrm{P}<0.01)$. Both the HF and DA surfaces had the lowest value of $F$. nucleatum adhesion with highly significant differences to all other surfaces $(\mathrm{P}<0.01)$ except with the AEHT surface.

Highly significant difference were found in PT surface $(\mathrm{P}=0.002)$ and significant differences in the DA surface $(\mathrm{P}=0.011)$ within each surface between the percentage of bacterial adhesion with and without saliva.

\section{Discussion}

Dental implant success is governed by the reaction between dental implant surface characteristics and the biological response to them. ${ }^{15}$ Altered surface characteristics have been reported to have potential effects on the outcome of dental implant therapy. ${ }^{10}$ It is well known that an increase in surface roughness leads to enhanced bacterial adhesion by providing shelter within surface irregularities. ${ }^{2}$ Quirynen and coworker ${ }^{16}$ have shown a clear relationship between plaque and surface roughness. Therefore, in order to be able to evaluate other surface characteristics, surface roughness was minimized by making the roughness less than $0.2 \mu \mathrm{m}$ as it has been stated previously that roughness less than $0.2 \mu \mathrm{m}$ will not have an effect on bacterial retention as most bacteria are larger in size. ${ }^{17,18,19}$

Interactions between implant and biological tissue depend on surface properties and not on the bulk of the material. ${ }^{20}$ Wide arrays of surfaces were included in this experiment with different modifications and different surface properties. 


\section{Surface characteristics}

The AN surface showed many pits on the surface in agreement with previous studies. ${ }^{21}$ These pits in a shape which supports the previous suggestion by Lausmaa and coworker ${ }^{22}$ that the growth of the oxide layer is due to ions coming out from inside the metal and not through deposited on it.

The DA surface showed a typical picture of an etched surface, with pits and scratches across the whole surface similar to the commercially available Osseotite implant (Osseotite implant, 3i palm Beach Gardens, FL) produced by dual acid etching surface modification. ${ }^{23}$

The strong impact of exposed grain structure on the titanium surface was seen on AEHT surface optical microscope image, in which different grains reflect different colours with a mosaic appearance. One explanation for these mosaic colours is that the oxide layer is first removed by alkali etching thus exposing the grain structure, then during heat treatment each grain reacts in a different way resulting in this different colour as has been stated previously ${ }^{24}$ that titanium surfaces with "poly crystalline" surfaces (surfaces with exposed grain) are more likely to cause different patterns of oxide growth and different oxide micro textures.

Another explanation is that the colour of the titanium oxide depends on light reflection and since each one of these grains has its own orientation and inclination they will reflect the colour differently according to the light ray strike and reflect angles.

A high level of surface wettability (hydrophilicity) has been considered previously to be one of the essential requirements for successful osseointegration. ${ }^{5}$ However, a recent study on one of the successful commercial implants (SLA from Straumann AG implant) revealed that a hydrophobic surface showed more cell attachment than other hydrophilic surfaces. ${ }^{25}$

The AEHT surface showed a hydrophobic surface characteristic which can be related to the presence of rutile crystalline oxide structure on the surface. However, Lewandowskae and coworker ${ }^{26}$ also showed an increase in the hydrophobicity of AEHT surfaces.

The AN surface which had an anatase crystalline oxide layer also showed an increase in hydrophobicity. This result disagrees with a previous study ${ }^{27}$ that showed that the titanium oxide formed by anodization significantly improves the hydrophilicity. It is thought that this disagreement may be due to the crystallinity of the oxide structure as the oxide structure was reported amorphous in this study.

The contact angle result of the HF surface showed a significant increase in hydrophobicity in agreement with Lamolle and coworkers ${ }^{28}$ who found that exposure of titanium surfaces to hydrofluoric acid led to an increase in surface hydrophobicity. They suggest that the roughness and presence of fluoride and oxide along with a low carbon level might be the cause of increased hydrophobicity. It has been thought that in addition to the above reasons it may also be due to grain exposure; as both the HF and DA surfaces had the same contact angle measurement $\left(104^{\circ}\right)$.

Despite heat treatment resulting in a significant increase in surface microhardness measurements, also it changed this surface to be more brittle. This was noted whilst conducting microhardness measurements as sometimes the surface layer crushed under the load, therefore requiring a repetition of the process.

The AN surface showed the lowest value for microhardness, the possible explanation could be related to the effect of anodization on surface topography that change the surface nature from a solid, compact polished surface to a surface with many pits. The decrease in microhardness has also been reported previously by Kim and coworkers ${ }^{27}$ when they measured the elastic modulus of an anodized titanium surface.

Titanium oxide can be both amorphous and crystalline; the crystalline structure exists as three types: rutile, anatase and brookite. ${ }^{27,29}$ All are different in terms of their physical properties.

Heating titanium to a high temperature lead to increase oxide layer thickness. ${ }^{30}$ However, both HT and AEHT surfaces exhibit a typically rutile oxide phase. The AN surface presented a typical anatase phase in agreement with previous research. ${ }^{20,21}$ However, no crystalline phase could be found for the HF, DA and PT surfaces, Lausmaa and coworker ${ }^{22}$ explained this by the lack of electron and x-ray diffraction patterns in a surface with a thin oxide thickness as the oxide surface does not have enough bulk to form a long-range crystalline order.

$\mathrm{X}$-ray photoelectron spectroscopy is an analysis technique for the outer 1-10 $\mathrm{nm}$ of the sample surface by soft X-rays (photon irradiation), these photons have a limited penetrating power in a solid. ${ }^{31}$ Surface contamination with different elements (usually with organic material) is something can not be avoided. This layer is dynamic and its composition changes depending on the type of environment surrounding these surfaces. $^{32}$

In addition to the major components small amount of impurities were present in the superficial layer. For the AN surface the presence of fluoride was expected as hydrofluoric acid had been added to the anodizing 
electrolyte solution aiming to incorporate this element in the surface. ${ }^{27}$ Incorporation of elements as a result of adsorption of ions from the electrolyte has also been mentioned previously. ${ }^{22}$ The presence of nitrogen was not expected. Sul and coworkers ${ }^{20}$ and Lausmaa and Kasemo ${ }^{33}$ also detected nitrogen in their anodized surface and explained its presence as a contaminating element, the source of nitrogen may also be explained by the fact that nitrogen exists in commercial titanium with $0.03 \mathrm{wt} \%^{34}$ and was not detected in polished titanium as its percentage is beyond detection. During anodization the oxide layer increased in thickness by elements coming from beneath the oxide/metal interface ${ }^{22}$ which brings the nitrogen to the surface topography.

One of the interesting findings was the presence of calcium on the AEHT surface; no clear explanation is apparent for the presence of calcium. However, Lewandowska and coworker ${ }^{26}$ also found calcium in their AEHT surface and they explained its presence due to the preparation procedure. However, no step within the preparation procedure in this study involved calcium.

\section{Bacteriology}

Dental implants have been widely used as an important treatment option in replacing missing teeth. Bacterial growth and plaque formation on implant surfaces is still one of the major reasons for inflammation and infection around dental implants. ${ }^{1}$ S. mitis was chosen as they are pioneer colonizer bacteria ${ }^{34}$ that prepare the favorable environment for later colonizers which may be pathogenic such as Fusobacterium species and Prevotella species ${ }^{1}$.

As the bacteria in this study have different cellular sizes, it should be recognized that comparison between percentages of different bacterial adhesion is not applicable as the percentage of area covered by bacteria was measured.

has been reported that anatase crystalline structures decrease bacterial adhesion more than the amorphous structure ${ }^{35}$ which is in agreement with the results of this experiment as the AN surface with anatase crystalline surface oxide had a lower percentage of $S$. mitis adhesion than PT, DA and HF surfaces with amorphous crystalline surface oxide layer.

In addition, both the AEHT and HT surfaces showed relatively the same percentage of S. mitis adhesion without saliva which could be explained by the fact that both of them had a rutile oxide crystalline structure, and had a higher percentage of bacterial adhesion than anatase oxide crystalline structure (anodized surface).

It seems that the crystalline phase has no effect on the percentage of S. mitis adhesion after coating with saliva as both AEHT and HT surfaces had a rutile crystalline oxide layer, but there were significant differences in percentage of S. mitis adhesion between them. This difference could not be correlated to the presence of calcium on the AEHT surface as Yoshinari and coworker ${ }^{36}$ found that calcium significantly enhance bacterial adhesion which is opposite from the results of this study, however, they used different bacterial species in their study.

Before these experiments it was thought that there will be no adhesion or a low percentage of $F$. nucleatum adhesion to these surfaces as they are pathogenic bacteria and they need a layer of initial bacteria to adhere to. ${ }^{35}$ However, surprisingly, these bacteria showed an unexpected ability to adhere to some of these surfaces. There was significantly high percentage of $F$. nucleatum adhesion to surfaces with crystalline phase which could be correlated to the organization of the titanium oxide layers in a specific crystalline form (anatase, rutile).

\section{Conclusions/Summary}

Physiochemical properties of titanium can be altered depending on the type of modification and each surface modification results in a specific effect on the titanium surface topography.

Most of the modifications to a titanium surface increase its hydrophobicity and decrease its surface free energy. Anodization, heat treatment, alkali etching plus heat treatment procedures lead to organization of titanium oxide layer in crystalline form (anatase, rutile).

Increased surface hydrophobicity along with decreased surface free energy leads to decrease bacterial adhesion to titanium surface after coating with saliva.

Pathogenic bacteria have an affinity to adhere to all modified titanium surfaces without the assistance or presence of a pioneer bacterial layer.

\section{Reference}

[1]. Grössner-Schreiber B, Griepentrog M, Haustein I., Müller W.D, Lange K.P, Briedigkeit H, Göbel U.B. Plaque formation on surface modified dental implants: An in vitro study. Clin Oral Implants Res. 2001; 12: 543-551.

[2]. Amoroso P.F, Adams R, Waters M \& Williams D. Titanium surface modification and its effect on the adherence of Porphyromonas gingivalis: an in vitro Study. Clin Oral Implants Res. 2006; 17: 633-637.

[3]. Chiesa R, Giavaresi G, Fini M. Sandrini E, Giordano C, Bianchi A, Giardino R. In vitro and in vivo performance of a novel surface treatment to enhance osseointegration of endosseous implants. Oral Surg Oral Med Oral Pathol Oral Radiol Endod. 2007; 103: 74556. 
[4]. Yoshinari M, Oda Y, Kato T, Okuda K. Influence of surface modifications to titanium on antibacterial activity in vitro. Biomaterials 2001; 22: 2043-2048.

[5]. He J, Zhou W, Zhou X. The anatase phase of nanotopography titania plays an important role on osteoblast cell morphology and proliferation. J Mater Sci Mater Med. 2008; 19: 3465-3472.

[6]. Mabbouxa F, Ponsonnet L, Morriera J, Jaffrezicb N, Barsottia O. Surface free energy and bacterial retention to saliva-coated dental implant materials-an in vitro study. Colloids Surf B Biointerfaces. 2004; 39: 199-205.

[7]. Albrektsson T, Wennerberg A. Oral implant surfaces: part 1 - review focusing on topographic and chemical properties of different surfaces and in vivo responses to them. Int J Prosthodont. 2004; 17: 536-43.

[8]. Worthington P. An introduction. In: Worthington P, Lang B \& Levelle W. Osseointegration in dentistry. Illinois: Quinstessenc Publishing; 1994. p 11-18.

[9]. LeGue' hennec L, Soueidan A, Layrolle P, Amouriq Y. Surface treatments of titanium dental implants for rapid osseointegration. Dent Mater 2007; 23: 844-54.

[10]. Xavier S, Carvalho P, Beloti M, Rosa A. Response of rat bone marrow cells to commercially pure titanium submitted to different surface treatments. J Dent. 2003; 31: 173-180.

[11]. Lee B, Kim Y, Shin J, Lee K. Surface modification by alkali and heat treatments in titanium alloys. J Biomed Mater Res. 2002, 61, 466-473.

[12]. Gandhi N. Bacterial adhesion to titanium surface modified with chlorhexidine. [thesis] university of Bristol; 2007.41 p.

[13]. Macdonald W, Campbell P, Fisher J, Wennerberg, A. Variation in Surface Texture Measurements. J Biomed Mater Res B Appl Biomater. 2004; 70: 262-269.

[14]. Torchinsky I, Rosenman G. Wettability Modification of Nanomaterials by Low-Energy Electron Flux. Nanoscale Res Lett. 2009; 4: 1209-1217.

[15]. Thomsen P, Larsson C, Ericson L. Structure of the interface between rabbit cortical bone and implants of gold, zirconium and titanium. J Mater Sci Mater Med. 1997; 8: 653- 665.

[16]. Quirynen M, Van der Mei H, Bollen C. Schotte, A, Marechal, M, Doornbusch, G.I, Naert, I, Busscher H.J, van Steenberghe D. An in vivo study of the influence of the surface roughness of implants on the microbiology of supra and subgingival plaque. J. Dent. Res. 1993; 72: 1304-9.

[17]. Bollen L, Lambrecht P, Quirynen M. Comparison of surface roughness of oral hard materials to the threshold surface roughness for bacterial plaque retention: a review of the literature. Dent Mater. 1997; 13: 258-269.

[18]. Quirynen M, Papaioannou W, Van Steenberghe D. Intra oral transmission and the colonization of oral hard surfaces. J. Periodontol 1996; 67: 986-93.

[19]. Grössner-Schreibe B, Teichmann J, Hannig M, Dörfer C, Wenderoth D.F, Ott S.J. Modified implant surfaces show different biofilm composition under in vivo conditions. Clin Oral Implants Res. 2009; 20: 817-826.

[20]. Sul Y.T, Johansson C.B, Jeong Y, Albrektsson T. The electrochemical oxide growth behaviour on titanium in acid and alkaline electrolytes. Med Eng Phys. 2001; 23: 329-346.

[21]. Sul Y.T, Johanssona C.B, Petronisc S, Krozer a, Jeong Y, Wennerberg A, Albrektsson T. Characteristics of the surface oxides on turned and electrochemically oxidized pure titanium implants up to dielectric breakdown: the oxide thickness, micropore configurations, surface roughness, crystal structure and chemical composition. Biomaterials, 2002; 23:491-501.

[22]. Lausmaa J, Kasemo B, Mattsson H, Odelius H. Multi-technique surface characterization of oxide films on electropolished and anodically oxidized titanium. Appl Surf Sci. 1990; 45: 189-200.

[23]. Szmukler-Moncler S, Perrin D, Ahossi V, Magnin G, Bernard J. Biological Properties of Acid Etched Titanium Implants: Effect of Sandblasting on Bone Anchorage. Appl Biomat. 2004; 68: 149-159.

[24]. Larsson C, Thomsen P, Lausmaa J, Rodahl M, Kasemo B, Ericson, L. Bone response to surface modified titanium implants: studies on electropolished implants with different oxide thicknesses and morphology. Biomaterials. 1994; 15: 1062-1074.

[25]. Le Guehennec L, Lopez-Heredia M, Enkel B, Weiss P, Amouriq, Y, Layrolle P. Osteoblastic cell behavior on different titanium implant surfaces. Acta Biomater. 2008; 4: 535-543.

[26]. Lewandowska M, Włodkowska M, Olkowski R, Roguska A, Polak B, Pisarek M, Lewandowska-Szumieł M. and Kurzydłowski K. J. Chemical Surface Modifications of Titanium Implants. Macromol Symp. 2007; 253: 115-121.

[27]. Kim H, Yang Y, Koh J, Lee K, Park S. Fabrication and Characterization of Functionally Graded Nano-Micro Porous Titanium Surface by Anodizing. J Biomed Mater Res B Appl Biomater. 2009; 88: 427-435.

[28]. Lamolle S, Monjo M, Rubert M, Haugen H.J, Lyngstadaas S.P, Ellingsen J.E. The effect of hydrofluoric acid treatment of titanium surface on nanostructural and chemical changes and the growth of MC3T3-E1 cells. Biomaterials. 2009; 30: 736-742.

[29]. Metlkos-Hukovic M, Ceraj-Ceric M, Zagreb P. Anodic oxidation of titanium: Mechanism of non stochiometric oxide formation. Surface technology. 1985; 24: 273-283.

[30]. Olin H, Aronsson B, Kasemo B, Lausmaa J, Rodahl M. Scanning tunneling microscopy of oxidized titanium surfaces in air. Ultramicroscopy; 1992; 42: 567-571.

[31]. Moulder J, Stickle W, Sobol P, Bomben K. Hand book of X-ray photoelectron spectroscopy. 1st ed USA: Perkin-Elmer corporation, 1992: p 25.

[32]. Lausmaa J. Surface spectroscopic characterization of titanium implants materials. J Electron Spectros Relat Phenomena. 1996; 81: 343361 .

[33]. Lausmaa J, Kasemo B. Surface spectroscopic characterization of titanium implant materials. Appl Surf Sci. 1990 ; $44: 133-146$.

[34]. Cisar J, Takahashi Y, Ruhl S, Donkerloot J, Sandberg A. Specific inhibition of bacterial adhesion: Observation from study of grampositive bacteria that intiate biofilm formation on the tooth surface . Adv Dent Res. 1997; 11: 168-175.

[35]. Del Curto, B, Brunella, M, Giordano, C, Pedeferri, M.P, Valtulina, V, Visai, L, Cigada, A. Decreased bacterial adhesion to surfacetreated titanium. Int J Artif Organs. 2005, 28, 718-730. (Abstract. PMID: 16049906).

[36]. Yoshinari, M, Oda, Y, Kato, T, Okuda, K, Hirayamab, A. Influence of surface modifications to titanium on oral bacterial adhesion in vitro Inc. J Biomed Mater Res. 2000, 52, 388-394. 


\begin{tabular}{|c|c|c|c|c|c|c|}
\hline \multicolumn{1}{|c|}{} & PT & AN & HT & AEHT & DA & HF \\
\hline \multirow{2}{*}{$\operatorname{Ra}$} & 0.04 & $0.15^{* *}$ & $0.16^{* *}$ & $0.13^{* *}$ & $0.12^{* *}$ & $\begin{array}{c}0.15^{* *} \\
\pm 0.007\end{array}$ \\
& \pm 0.004 & \pm 0.011 & \pm 0.008 & \pm 0.013 & \pm 0.009 & \pm 09 \\
\hline \multirow{2}{*}{ Microhardness } & 169 & 166 & $291^{* *}$ & $192^{*}$ & $193^{*}$ & 168 \\
& \pm 4.78 & \pm 1.51 & \pm 45.75 & \pm 4.86 & \pm 5.00 & \pm 1.73 \\
\hline
\end{tabular}

(*) Significant difference with polished titanium surface $(\mathbb{R}<0.05)$

(*) Highly significant difference rith polished titanium surface $(\mathrm{R}: 0.01)$

Table 1- Surface roughness measurement $(\mathrm{Ra}$ in $\mu \mathrm{m})$ using non contact profilometer $(\mathrm{n}=10),(\mathrm{Ra}$ : the arithmetic mean of the absolute values of the surface point departures from the mean plane within the sampling area). The microhardness results in Vickers point number, the values represent the mean \pm standard error.

\begin{tabular}{|c|c|c|c|c|}
\hline & \multirow{2}{*}{ Contact angle } & \multirow{2}{*}{ SFE } & \multicolumn{2}{|c|}{ SFE } \\
\cline { 4 - 5 } & & & Disperse & Polar \\
\hline PT & $74.32 \pm 1.34$ & $37.58 \pm 0.67$ & $32.39 \pm 0.47$ & $5.19 \pm 0.21$ \\
\hline AN & $84.73^{* *} \pm 1.61$ & $38.59 \pm 0.7$ & $36.26 \pm 0.49$ & $2.32 \pm 0.2$ \\
\hline HT & $76.21 \pm 2.07$ & $35.72 \pm 1.32$ & $29.57 \pm 0.98$ & $6.15 \pm 0.46$ \\
\hline AEHT & $90.13^{* *} \pm 3.86$ & $35.11 \pm 1.36$ & $33.05 \pm 0.98$ & $2.06 \pm 0.38$ \\
\hline DA & $104.32^{* *} \pm 1.66$ & $31.61 \pm 1.07$ & $31.41 \pm 0.99$ & $0.2 \pm 0.04$ \\
\hline HF & $104.22^{* *} \pm 2.05$ & $32.35 \pm 1.03$ & $32.27 \pm 0.98$ & $0.08 \pm 0.02$ \\
\hline
\end{tabular}

(*) Highly significant difference with polished titanium surface ( $\mathrm{P}<0.01)$

Table 2- Average contact angle measurements (in $\theta$ ) using the sessile drop technique $(\mathrm{n}=10)$. The surface free energy (in $\mathrm{mN} / \mathrm{m}$ ) is shown with disperse and polar parts. The values represent the mean \pm standard error 


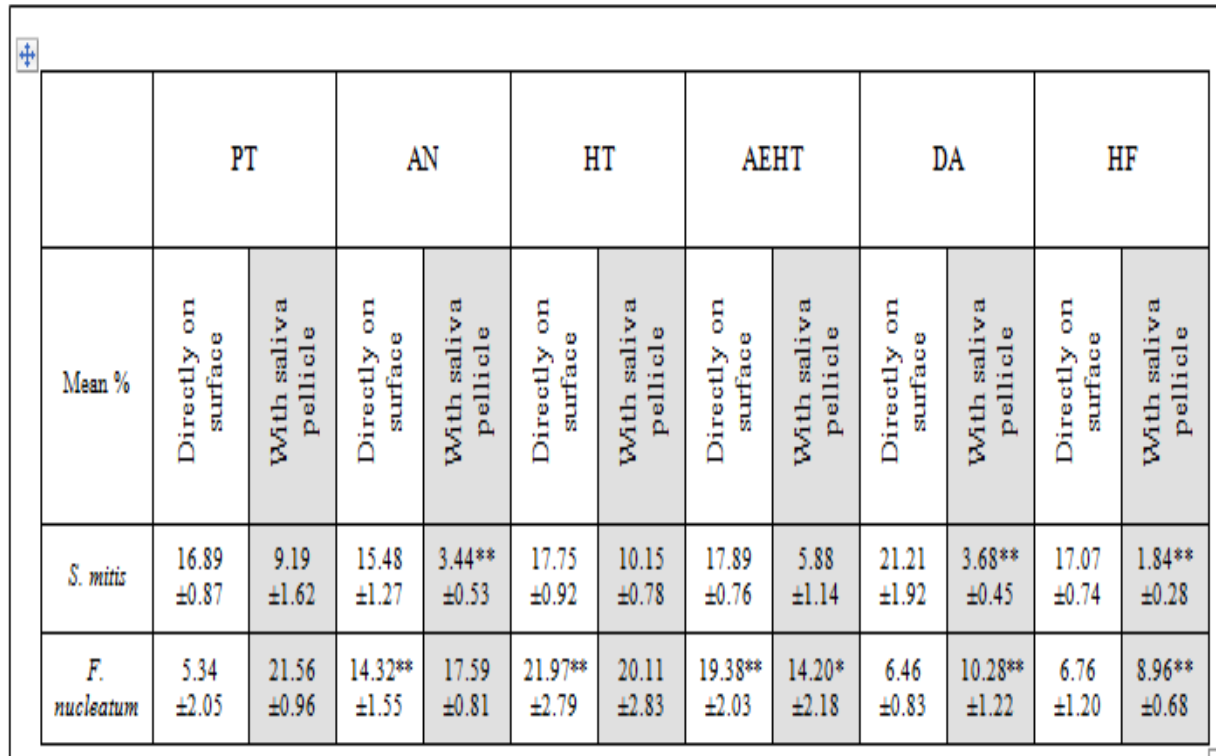

(•) Siggificant difference vith polished titanium surface $(\mathbb{P}<0.05)$

$(*)$ Highly siggificant difference with polished titanium surface $(\mathbb{R}<0.01)$

Table 3- Mean percentage of $S$. mitis and $F$. mucleatum bacterial adhesion to different modified titanium surfaces with and with out saliva coating $(\mathrm{n}=10)$. The values represent the mean \pm standard error.

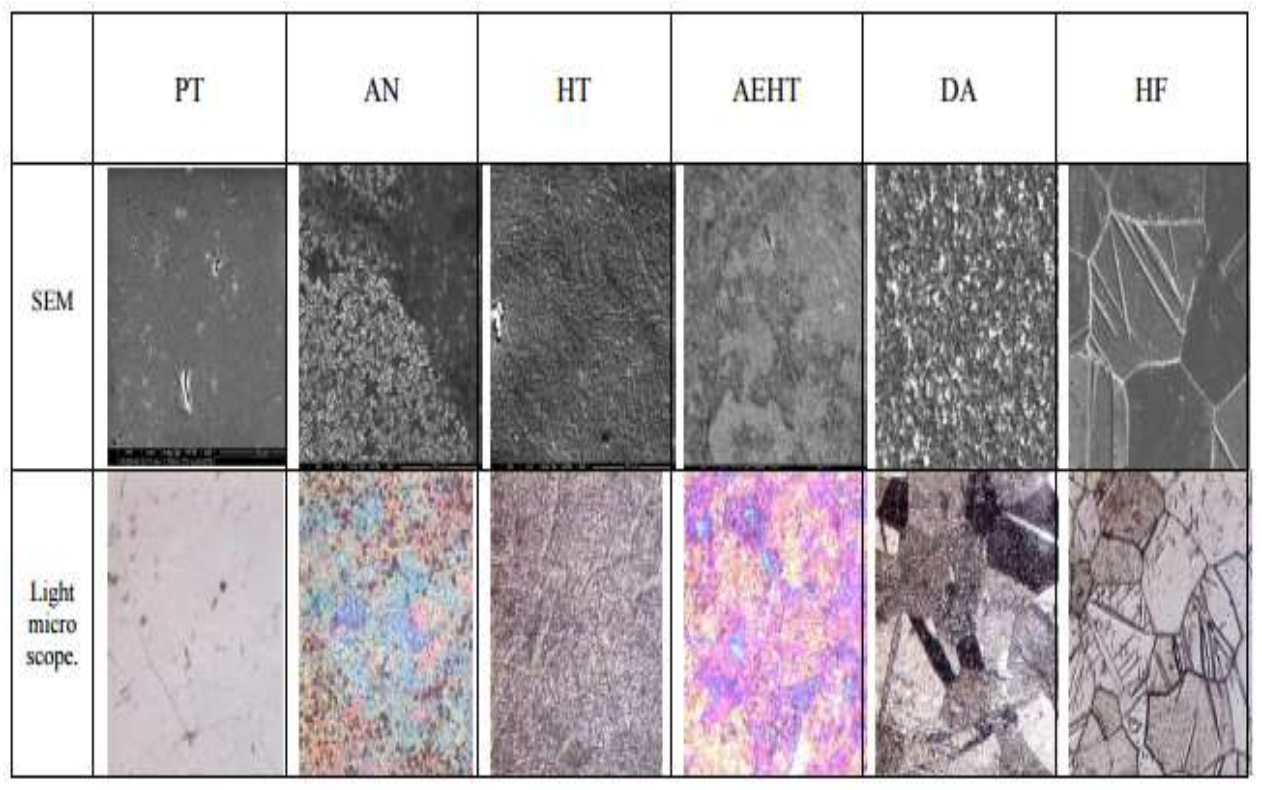

Figure 1.- Morphological assessment of different modified titanium surfaces using scanning electron microscope ( 750 magnification) and light microscope (50 magnification) 